\title{
Estimation of the standard deviation in three-dimensional microscopy by spatial statistics
}

\author{
L. M. SANCHEZ-BREA* \& E. BERNABEU† \\ *Departamento de Optica, Universidad Complutense de Madrid, Escuela Universitaria de Optica, Arcos \\ del Jalón s/n. 28037 Madrid, Spain \\ †Departamento de Optica, Universidad Complutense de Madrid, Facultad de Ciencias Físicas, Ciudad \\ Universitaria s/n. 28040 Madrid, Spain
}

Key words. Calibration, spatial statistics, standard deviation, three-dimensional microscopy, variogram.

\section{Summary}

Usually, the calibration process for three-dimensional microscopy involves the use of a reference flat surface. The random fluctuations of the topographic image for this reference surface are used for determining the uncertainty of the microscope. When the sample material or the measuring conditions of the microscope are modified (such as the objective used in a confocal microscope, or the tip in an atomic force microscope), the measuring conditions vary and thus a new calibration is required. In this work, a technique based on spatial statistics methods (more specifically, the variogram function) is proposed to determine accurately the standard deviation for threedimensional microscopy that does not require a reference flat surface and therefore eliminates the need for a previous calibration process of this parameter.

\section{Introduction}

In three-dimensional (3D) microscopy, such as scanning probe microscopy (SPM) and confocal microscopy (CM), the final result is a topographic image $Z(x, y)$, which, as with every measurement process, is affected by random fluctuations, which can preclude a faithful representation of the objects, when microscopy is used for visualizing (Conan et al., 1992). Thus, the image is usually filtered, using a number of techniques such as linear, median and adaptive filtering (Pratt, 1978). The topographic images of 3D microscopy are frequently used to calculate dimensional parameters of the object (for example, the radius of curvature, the height of a step, etc.) and therefore

Correspondence to: Dr L. M. Sanchez-Brea. Tel.: + 34913944555 ; fax: + 3491 394 4674; e-mail: sanchezbrea@fis.ucm.es the uncertainty of the heights needs to be determined for calculating the standard error of the dimensional parameters.

For a quantity without spatial dependence $Q$, the usual technique for determining the standard error is to perform a calibration first, obtaining a number of measurements under the same conditions, and calculating the standard error $(u)$ from the standard deviation (s) (ISO, 1995). When several measurements $\left(Q_{i}, i=1, \ldots, N\right)$ are performed, a certain randomness around the correct value is expected (random variable), and the most accurate prediction of $Q$ is the arithmetic mean $\langle Q\rangle_{i}$ where $\langle\cdot\rangle_{i}$ means average with respect to $i$. The dispersion of the magnitude is determined by the experimental variance $s^{2}$ (ISO, 1995). The square root of this quantity is the well known experimental standard deviation, $s(Q)$, standard deviation for short (Bevington, 1969), and the standard error is obtained from the standard deviation of a random variable by $u(\underline{Q})=s(\underline{Q}) / \sqrt{N}$.

When the measured quantity presents a spatial dependence, as in topographical images, the result of the measurement consists of a number of data at different locations, $Z\left(\mathbf{x}_{i, j}\right)$, where $i=1, \ldots, N, j=1, \ldots, M$ represent the position. Normally in imaging and microscopy, data are equidistantly distributed and then $\mathbf{x}_{i, j}=(i \Delta x, j \Delta y)$, where $\Delta x$ and $\Delta y$ are the distances between consecutive data in the $x$ and $y$ axis, respectively. To determine the standard deviation, several $(K)$ 3D images should be obtained, being the experimental variance at each position

$$
s^{2}\left[Z\left(\mathbf{x}_{i, j}\right)\right]=\frac{1}{K-1} \sum_{k=1}^{K}\left[Z_{k}\left(\mathbf{x}_{i, j}\right)-\left\langle Z\left(\mathbf{x}_{i, j}\right)\right\rangle_{k}\right]^{2}
$$

where $\mathbf{x}_{i, j}$ are vectors and $k$ stands for each image.

A more common approach to determine the standard deviation for 3D microscopy is using a reference flat surface, as only one image is required. The variations in the experimental 
data are assumed to be due only to random fluctuations, not to variations in the topography. In this case, the variance is

$$
s^{2}(Z)=\frac{1}{N M-1} \sum_{i=1}^{N} \sum_{j=1}^{M}\left[Z\left(\mathbf{x}_{i, j}\right)-Z_{0}\right]^{2}
$$

where

$$
Z_{0}=\frac{1}{N M} \sum_{i=1}^{N} \sum_{j=1}^{M} Z\left(\mathbf{x}_{i, j}\right)
$$

The variations in the height of the flat surface should be much lower than the standard deviation obtained, because small variations in the topography may increase the calculated standard deviation considerably.

The measuring conditions of the microscopes may vary with time, making a periodical recalibration necessary. A calibration of the standard deviation is also required when the device is modified, for example, changing the objective in CM, or the probe in AFM. In addition, the measuring conditions in 3D microscopy may depend on the sample to be measured. For example, in AFM, the interaction forces depend on the material, and in CM the quality in the reconstruction of the topography is influenced by the intensity of the reflected light, which also depends on the material of the sample. Therefore, the material of the reference flat surface and that of the sample should be the same. If not, the standard deviation computed for the flat surface could be not suitable for the sample. This would mean having one reference surface for each material to be measured.

In this work, a technique, based on spatial statistics, is proposed in order to determine the standard deviation for a 3D microscope with only one non-uniform topographical image. As a consequence, a previous calibration of this parameter is not required, as it can be easily determined for each image. The technique has been applied to 3D images obtained with AFM and CM.

\section{Estimation of the standard deviation using the variogram}

To determine the standard deviation using a non-flat surface, the spatial correlation of the topography will be used. For this, the variogram function (Cressie, 1991) has been used, which is defined as

$$
2 \gamma(\mathbf{h})=\left\langle[Z(\mathbf{x}+\mathbf{h})-Z(\mathbf{x})]^{2}\right\rangle_{x}
$$

where $\mathbf{h}$ is a vector, and $\langle\cdot\rangle_{\mathbf{x}}$ means averaging with respect to $\mathbf{x}$. Let us obtain $K$ images of the same topography, regularly sampled at locations $\mathbf{x}_{i, j}=(i \Delta x, j \Delta y)$, where $i=1, \ldots, N, j=1, \ldots, M$, $\Delta x$ and $\Delta y$ are the distances between data in the $x$ and $y$ axis, respectively (Fig. 1). Then the value of the pixel $(i, j)$ for the image $k$ is denoted by $Z_{k}\left(x_{i, j}\right)$. An estimation of the variogram for such case is

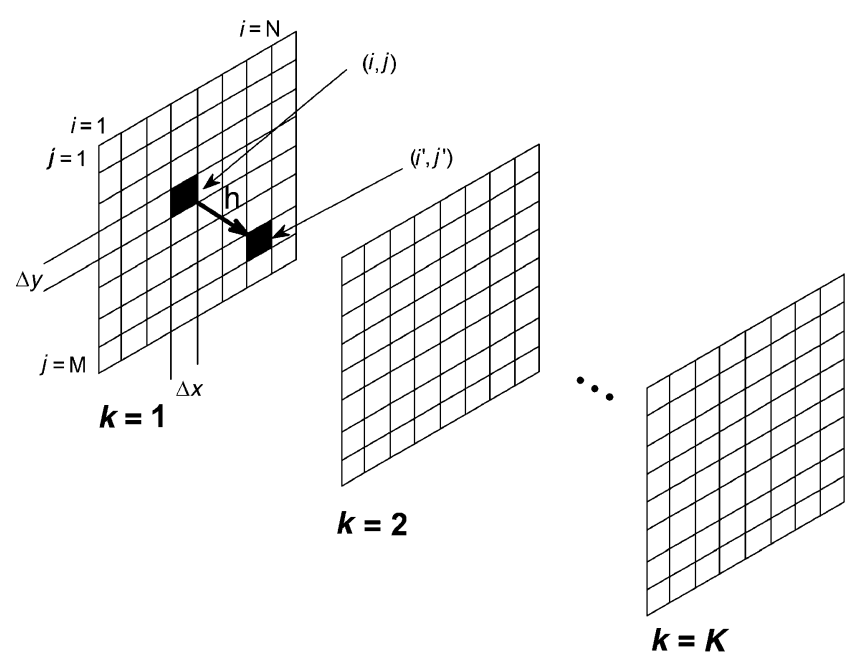

Fig. 1. Sketch representing the different topographical images obtained and the pixels of each image.

$$
2 \gamma(\mathbf{h})=\frac{1}{N(\mathbf{h})} \sum_{\substack{N(\mathbf{h}) \\ \mathbf{h}=\mathbf{x}_{i, f^{\prime}} \mathbf{x}_{i, j}}}\left[Z_{k^{\prime}}\left(\mathbf{x}_{i^{\prime}, j^{\prime}}\right)-Z_{k}\left(\mathbf{x}_{i, j}\right)\right]^{2}
$$

where $N(\mathbf{h})$ is the number of pairs $\left[Z_{k}\left(x_{i, j}\right), Z_{k^{\prime}}\left(x_{i^{\prime}, j^{\prime}}\right)\right]$ whose vector displacement is $\mathbf{h}=\mathbf{x}_{i^{\prime}, j^{\prime}}-\mathbf{x}_{i, j}=\left[\left(i^{\prime}-i\right) \Delta x,\left(j^{\prime}-j\right) \Delta y\right]$. The sum in Eq. (4) is made over $i, i^{\prime}, j, j^{\prime}, k, k^{\prime}$. As shown in SanchezBrea \& Bernabeu (2002), the value of the semivariogram at the origin $\gamma(0)$ is the average of the variances at all positions $x_{i, j}$

$$
\gamma(\mathbf{0})=\left\langle s^{2}\left[Z\left(\mathbf{x}_{i, j}\right)\right]\right\rangle_{i, j}=\frac{1}{N M} \sum_{i=1}^{N} \sum_{j=1}^{M} s^{2}\left[Z\left(\mathbf{x}_{i, j}\right)\right]
$$

where $\langle\cdot\rangle_{i, j}$ means averaging with respect to $i$ and $j$, and $s^{2}\left[Z\left(\mathbf{x}_{i, j}\right)\right]$ is the variance of the heights $Z$ at each location $x_{i, j}$ and it is defined as in Eq. (1). Therefore, when $s^{2}\left[Z\left(\mathbf{x}_{i, j}\right)\right]$ is assumed to be independent on the position the standard deviation of the heights $Z$ at a given location $\mathbf{x}_{i, j}$ due to the random fluctuations is given by

$$
s_{\gamma}=\sqrt{\gamma(0)}
$$

Our interest is to determine the standard deviation with only one topographical image $(K=1)$. Then, $\gamma(0)$ cannot be computed as there are not pairs $\left[Z_{k}\left(x_{i, j}\right), Z_{k^{\prime}}\left(x_{i^{\prime}, j}\right)\right]$ and Eq. (1) is not well defined. This inconvenience can be overcome by extrapolating the semivariogram

$$
s_{\gamma} \approx \lim _{\mathbf{h} \rightarrow 0} \sqrt{\gamma(\mathbf{h})}
$$

This extrapolation may be performed in several ways (Christiensen, 1985; Cressie, 1991), such as a minimal squares fitting. In most samples measured, the first data of $\sqrt{\gamma(\mathbf{h})}$ fits well to a straight line, being then possible to perform a linear 
extrapolation for determining $s_{\gamma}$. This procedure is not very time consuming and the error when computing $s_{\gamma}$ can be analytically obtained, resulting in

$$
e \approx b \Delta h / \sqrt{3}
$$

where $b$ is the slope of the fitting and $\Delta h$ is the distance between two adjacent points of the discrete variogram. That means that the error in the estimation of $s_{\gamma}$ decreases with high magnifications and using flat profiles. A quick and easy technique for implementing the linear extrapolation is to consider only the first two points of the variogram (at $\Delta h$ and $2 \Delta h$ ). In this case, the standard deviation is determined by

$$
s_{\gamma} \approx 2 \sqrt{\gamma(\Delta h)}-\sqrt{\gamma(2 \Delta h)}
$$

When the first data of the variogram cannot be approximated to a straight line, then other functions can be used for the fitting, but then the error in the standard deviation proposed in Eq. (8) is not valid and it has to be obtained for each case.

\section{Application to 3D microscopy}

The proposed technique has been applied to 3D images obtained by confocal microscopy (CM) and atomic force microscopy (AFM). A Burleigh's ARIS 3300 atomic force microscope (Burleigh Instruments Inc, Fishers, New York) and a Sensofar Tech's PL confocal imaging profiler (Sensofar Tech, Barcelona) have been used.

The standard calibration procedure [Eq. (2)] has been performed using a $3 \times 3 \mathrm{~cm}^{2}$ reference flat surface with a nominal maximum variation in height of $10 \mathrm{~nm}$. This variation is long range and, thus, it is much lower for the measured area $(150 \times 150 \mu \mathrm{m})$. If it is considered that the long range variation is approximately linear with the length, the maximum variation in height is around $0.1 \mathrm{~nm}$ at the measured area (we think that it will be larger, but no more than $1 \mathrm{~nm}$ ). Nevertheless, the reference flat surface that has been used presents several surface defects (with a vertical size of around $40 \mathrm{~nm}$ that may contaminate the determination of the standard deviation (Fig. 2a).

The standard deviation calculated with the standard technique for this reference surface was $s=6.2 \mathrm{~nm}$ for the AFM. This flat surface was also measured with the $\mathrm{CM}$, using three optical objectives: $20 \times, 50 \times$ and $100 \times$. The results for the standard technique were $s=11.5 \mathrm{~nm}, 6.1 \mathrm{~nm}$ and $2.0 \mathrm{~nm}$, respectively. As an example, in Fig. 2(b), the histogram obtained for the case of $100 \times$ is shown.

The standard deviation for this reference flat surface has also been computed using the variogram [Eq. (7)]. In principle, the determination of the variogram can be performed with the complete data set of the image. However, the computing time is usually prohibitive. As a consequence, the use of a small part of the image, such a 2D profile, was preferred. Any
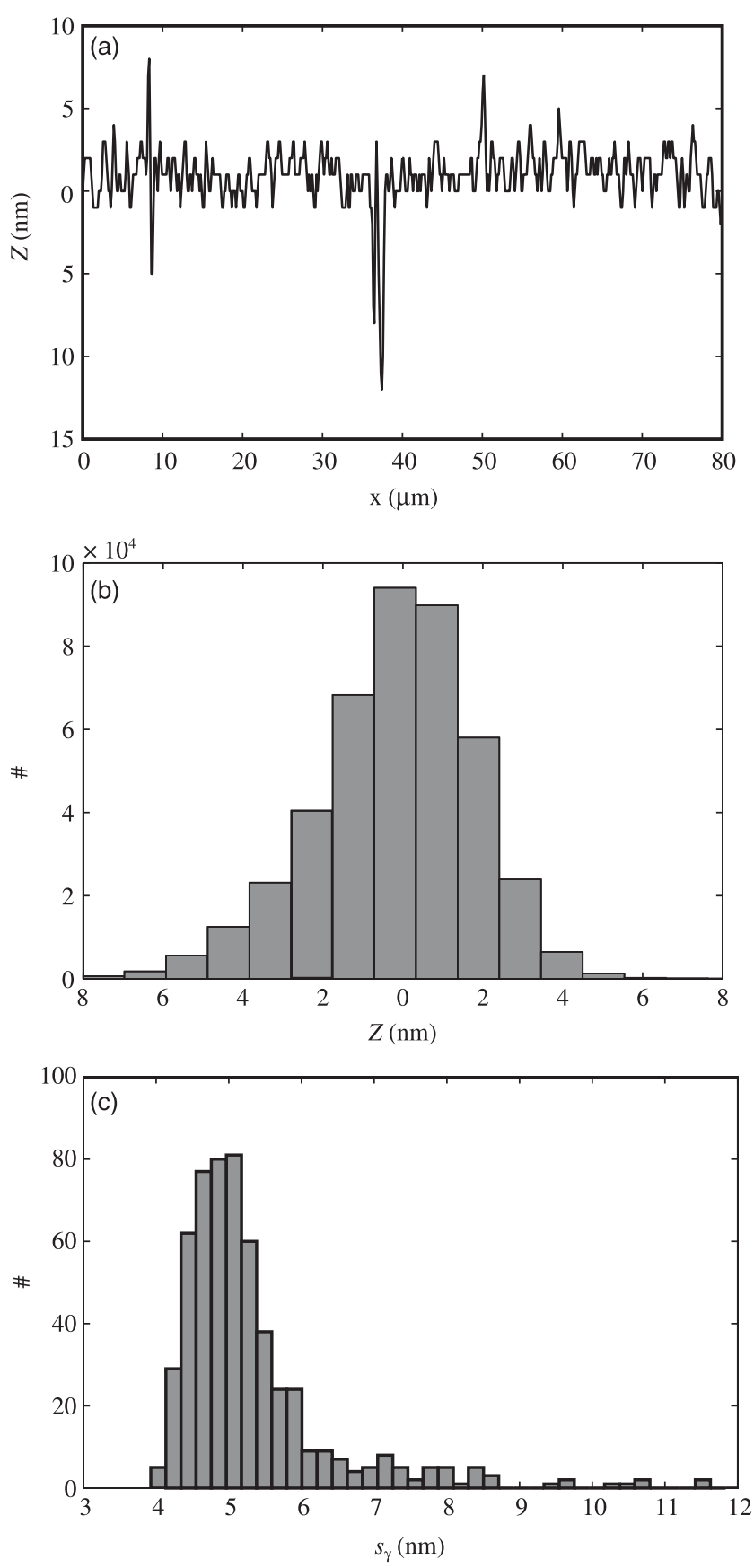

Fig. 2. (a) Profile of a reference flat surface obtained with a Sensofar's PL $\mu$ Confocal Imaging Profiler (objective 100x). A surface defect (hole with a depth of $10 \mathrm{~nm}$ ) is detected. (b) Histogram of the heights measured for this reference flat surface. The standard deviation of the heights $s$ obtained with the standard technique is $2.0 \mathrm{~nm}$. (c) Histogram of the standard deviations $s_{\gamma}$ computed with the variogram for 500 random profiles (objective $50 \times$ ).

profile is valid, although there may be certain variability in the determination of the standard deviation. Five hundred random profiles with a length between 50 and 400 points have been used. The mean standard deviation computed with the 

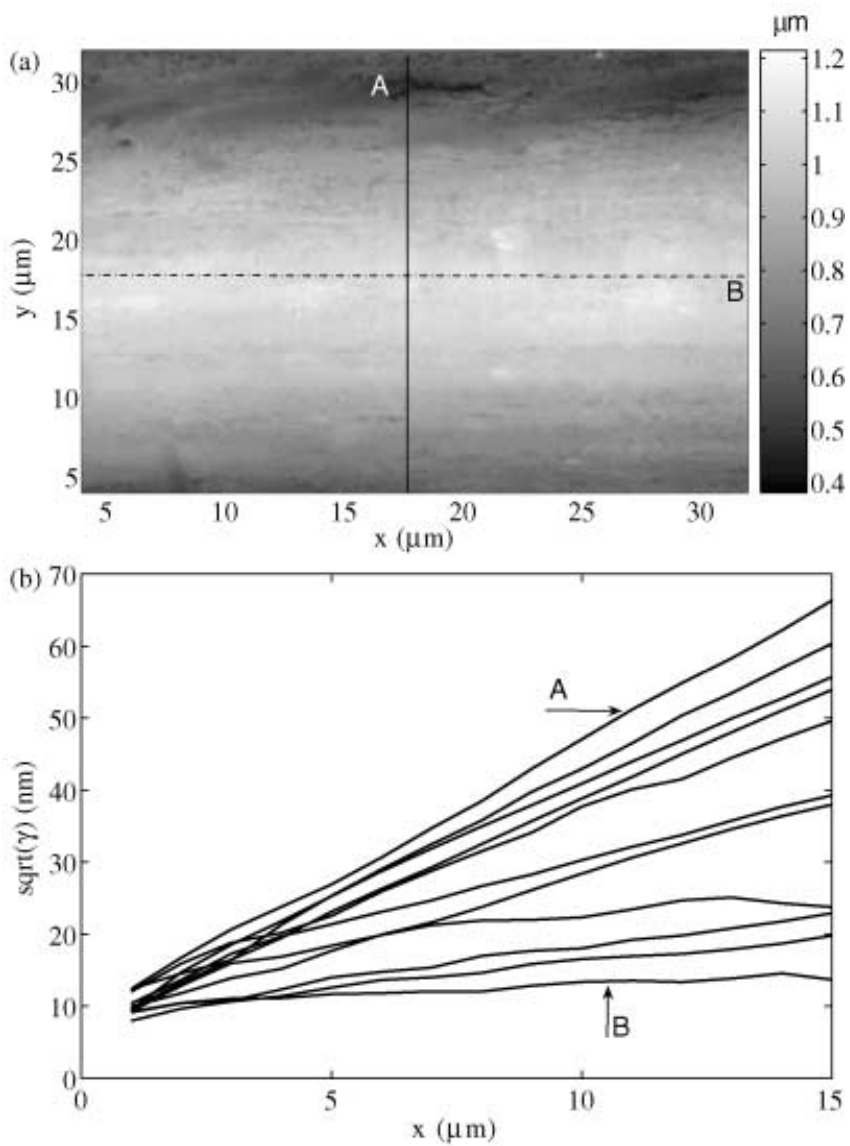

Fig. 3. (a) 3D topographic image of a steel wire with a diameter of a diameter of $300 \mu \mathrm{m}$ obtained with a Burleigh's ARIS 3300 AFM. (b) Variogram for several profiles. It is clear that $\sqrt{ } \gamma$ is approximately linear and therefore a linear extrapolation can be used for computing $s_{\gamma}$. Profiles A and B are shown in (a) and are those with a highest and lowest slope. For computing $s_{\gamma}$, Profile B is better than Profile A, as the error in the estimation is lower.

variogram resulted in $s_{\gamma}=10.2 \mathrm{~nm}, 5.4 \mathrm{~nm}$ and $1.3 \mathrm{~nm}$ for the $20 \times, 50 \times$ and $100 \times$, respectively, using the extrapolation of Eq. (9). These results are in accordance with those obtained by the standard technique. As an example, in Fig. 2(c) the histogram for the computation of $s_{\gamma}$ for the $50 \times$ objective is shown. A linear fitting to the variogram produced similar results. The variability in the determination of $s_{\gamma}$ was measured using the standard deviation of these 500 random profiles, resulting in $1.3 \mathrm{~nm}, 1.4 \mathrm{~nm}$ and $0.2 \mathrm{~nm}$, respectively. For the AFM, the value of $s_{\gamma}$ for the reference flat surface was $s_{\gamma}=5.6 \mathrm{~nm}$.

The value of $s_{\gamma}$ for the four cases (CM with three microscope objectives and AFM) was lower to that obtained with the standard technique. We think that it is due to the small surface defects of the reference flat surface, that strongly affect the standard technique. However, the value of $s_{\gamma}$ computed with the variogram is not affected by these defects. This is clearly shown when the standard deviation is computed for selected
2D profiles which do not pass over the surface defects, as the standard deviation computed with Eq. (2) is very close to the value obtained with Eq. (7).

The variogram technique has also been applied to non-flat surfaces. As an example, the 3D topography of a steel wire with a diameter of $300 \mu \mathrm{m}$, obtained with the AFM is shown in Fig. 3(a). The conditions for the microscope were the same as those for the measurement of the reference flat surface. The value of $s_{\gamma}$ for 1000 random $2 \mathrm{D}$ profiles with a length between 50 and 400 points has been determined. In Fig. 3(b) it is clearly shown that $\sqrt{ }$ is approximately linear. The mean value of $s_{\gamma}$ for these 1000 profiles was $s_{\gamma}=7.53 \mathrm{~nm}$, with the standard deviation $1.9 \mathrm{~nm}$. These results are in accordance to that obtained with the flat surface. The variability is due to the strong differences of the profiles. As it has been mentioned before, the best results are obtained with profiles of flat surfaces (profile B).

Also, several samples have been measured with CM, giving results in accordance with those obtained with the standard technique. As an example, a sample consisting of a diffraction grating with a nominal period of $40 \mu \mathrm{m}$ and a nominal height of $240 \mathrm{~nm}$ is shown in Fig. 4(a). The microscope objective was $100 \times$. As a first step, $s_{\gamma}$ has been computed using the complete data set resulting $s_{\gamma}=2.09 \mathrm{~nm}$. To see that the 2D profiles are valid for determining $s_{\gamma}, 1000$ random profiles with lengths between 50 and 300 points have been used (Fig. 4b). The mean value for these profiles resulted in $s_{\gamma}=2.30 \mathrm{~nm}$. The variability in the estimation of $s_{\gamma}$ for these 1000 samples resulted $0.5 \mathrm{~nm}$. We have also obtained $s_{\gamma}$ by means of a linear interpolation of the first 10 points (using the same 1000 computations of the former approach). The mean standard deviation computed for these cases was $s_{\gamma}=2.25 \mathrm{~nm}$, with the variability for these data being $0.3 \mathrm{~nm}$.

\section{Conclusions}

In this work, a technique for estimating the standard deviation in 3D microscopy by means of spatial statistics has been shown. This technique consists of estimating the variogram at the origin, which is equal to the variance of the random fluctuations. When only one image is used, the variogram at the origin is not well defined. Thus, an extrapolation of the variogram function is required. The technique has been applied to 3D images obtained with CM and AFM. The experimental results achieved with this new technique are in good agreement to those obtained using a reference surface. In Table 1, a comparison of the standard technique and the variogram technique is presented, for flat and non-flat surfaces.

Since the standard deviation can be easily computed for each topographic image using the variogram, a previous calibration, using flat reference surfaces, is not longer necessary. Also, a better estimation of the standard deviation is obtained with the variogram even when reference flat surfaces are used, since the surface defects that it may present do not effect 

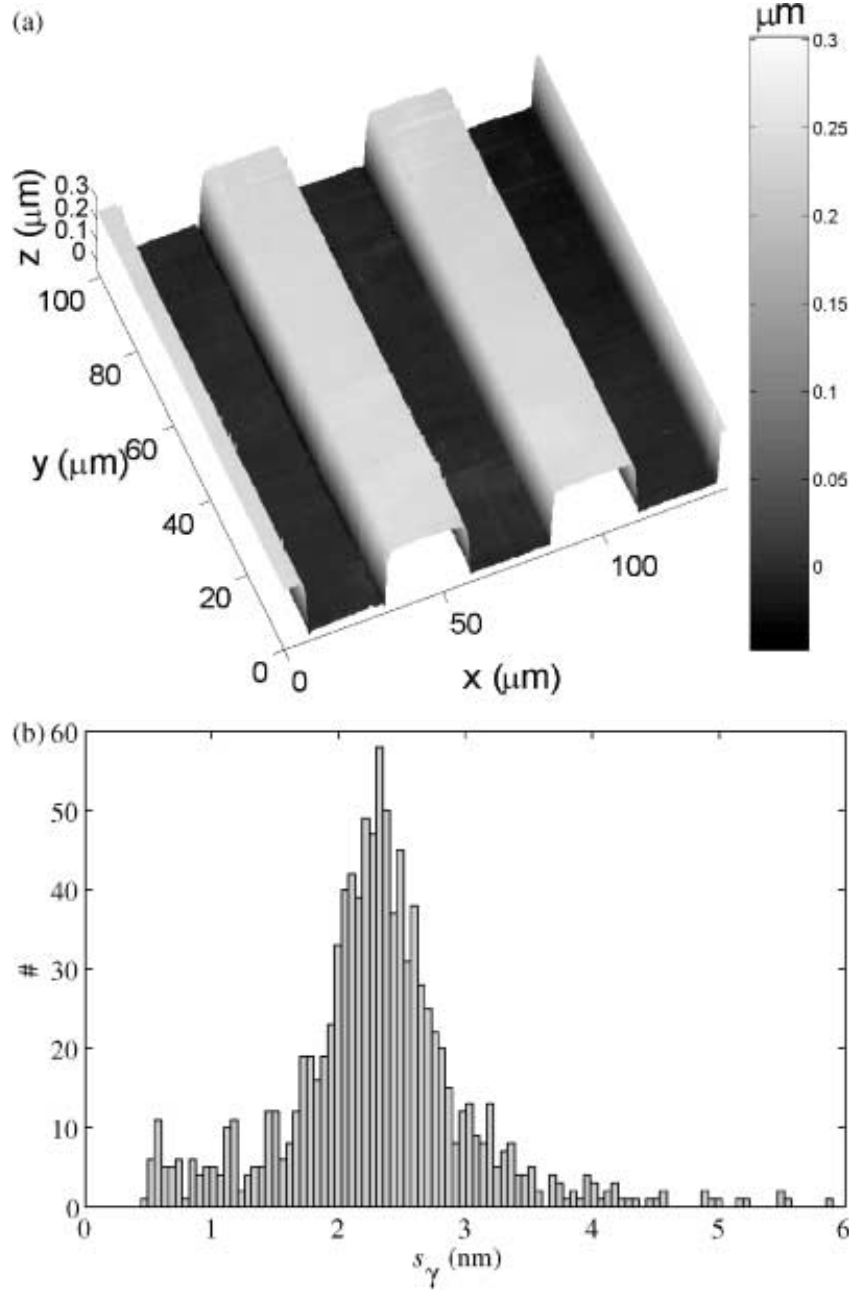

Fig. 4. (a) 3D topographic image of a diffraction grating with a period of $20 \mu \mathrm{m}$ and a height of $240 \mathrm{~nm}$ obtained with the confocal microscope (objective 100×). (b) Histogram of the standard deviations $s_{\gamma}$ computed with the variogram for 1000 random profiles.

the calculation of $s_{\gamma}$, whereas the standard technique is quite affected by such surface defects. On the other hand, our proposed method presents a higher workload of computations, whereas the standard deviation according to the standard method may be obtained with a table calculator. One draw-
Table 1. Comparison between the standard technique, using a reference flat surface, and the variogram technique for computing the standard deviation of the heights in 3D microscopy

\begin{tabular}{|c|c|c|c|}
\hline & \multicolumn{2}{|c|}{ Flat surface } & \multirow{2}{*}{$\frac{\text { Non-flat surface }}{s_{\gamma}}$} \\
\hline & $s$ & $s_{\gamma}$ & \\
\hline CM $20 \times$ & 11.5 & 10.2 & 11.6 \\
\hline CM 50x & 6.1 & 5.4 & 5.3 \\
\hline CM 100× & 2.0 & 1.3 & 2.3 \\
\hline AFM & 6.2 & 5.5 & 7.53 \\
\hline
\end{tabular}

back of our method is that there is a need to know previously the functional dependence of the variogram at the origin. Further tests are required to evaluate the new method in routine applications to different objects.

\section{Acknowledgements}

The authors thank Javier Alda for his fruitful discussions and interest in this research. This work has been supported by the Secretaría de Estado de Política Científica y Tecnológica (Spain), project DPI2001-1238. Dr Sanchez-Brea is currently contracted within the frame of the 'Ramón y Cajal' research program of the Ministerio de Educación y Ciencia of Spain.

\section{References}

Bevington, P. (1969) Data Reduction and Error Analysis for the Physical Sciences. McGraw-Hill, New York.

Christiensen, R. (1985) Linear Models for Multivariate, Time Series, and Spatial Data. Springer-Verlag, Berlin.

Conan, V., Gesbert, S., Howard, C.V., Jeulin, D., Meyer, F. \& Renard, D. (1992) Geostatistical and morphological methods applied to 3dimensional microscopy. J. Microsc. 166, 169-184.

Cressie, N.A. (1991) Statistics for Spatial Data. J. Wiley \& Sons, New York. ISO (1995) Guide to the Expression of the Uncertainty in Measurement. International Standardization Organization, Geneva.

Pratt, W.K. (1978) Digital Image Processing. J. Wiley \& Sons, New York. Sanchez-Brea, L.M. \& Bernabeu, E. (2002) On the standard deviation in CCD cameras: a variogram-based technique for non-uniform images. J. Electron. Imag. 11, 121-126. 
Copyright of Journal of Microscopy is the property of Blackwell Publishing Limited and its content may not be copied or emailed to multiple sites or posted to a listserv without the copyright holder's express written permission. However, users may print, download, or email articles for individual use. 